\title{
Transformation temperatures, elastic and anelastic properties of Cu-Al-Ni crystals subjected to impact loading
}

\author{
S. Golyandin ${ }^{1}$, S. Kustov ${ }^{1,2}$, S. Nikanorov ${ }^{1}$, K. Sapozhnikov ${ }^{1}$, A. Sinani ${ }^{1}$, \\ J. Van Humbeeck ${ }^{3}$, R. Schaller ${ }^{4}$ and R. De Batist ${ }^{5}$ \\ ${ }^{1}$ A.F. Ioffe Physical-Technical Institute, Politekhnicheskaya 26, 194021 St. Petersburg, Russia \\ ${ }^{2}$ LMPM, ENSMA, BP. 109, Chasseneuil-du-Poitou, 86960 Futuroscope, France \\ ${ }^{3}$ Katholieke Universiteit Leuven, Departement MTM, De Croylaan 2, 3001 Heverlee, Belgium \\ ${ }^{4}$ Institut Génie Atomique, École Polytechnique Fédérale de Lausanne, 1015 Lausanne, \\ Switzerland \\ ${ }^{5}$ University of Antwerpen, RUCA, IMS, Middelheimlaan 1, 2020 Antwerpen, Belgium
}

\begin{abstract}
Experimental investigations of the influence of impact loading on properties of $\mathrm{Cu}-\mathrm{Al}-\mathrm{Ni}$ single crystals have been performed. Crystals in the $\beta$, phase were impacted with pulses of a uniaxial plane strain wave with a duration of about $2 \times 10^{-6}$ s. A normal component of stress in the direction of the pulse propagation ranged from 0.5 to $5.4 \mathrm{GPa}$. For as-quenched and impacted crystals martensitic transformation temperatures were determined, the Young's modulus (YM), strain amplitudeindependent and strain amplitude-dependent internal friction were measured at a frequency of about $100 \mathrm{kHz}$ over the temperature range of $300-90 \mathrm{~K}$ for strain amplitudes of $10^{-7}-2 \times 10^{-4}$. Experimental results indicate that $\beta_{1} \rightarrow \gamma_{1}^{\prime}$ martensitic transformation (MT) and plastic deformation of the martensite are induced by the impact. The impact loading generates a "structure memory effect»: the $\mathrm{YM}$ in the austenite is not sensitive to the impact, but the consequent temperature-induced MT reveals a dramatic influence of the impact on the $\mathrm{YM}$ of the $\gamma_{1}{ }^{\prime}$ martensite. The conclusion is drawn that the observed effect is fundamentally similar to the two-way memory effect, but is extremely sensitive to the impact stress. The origin of this phenomenon is attributed to the internal stresses, created by impact, which control the nucieation of preferentially oriented anisotropic martensitic variants during temperature-induced MT in impacted crystals. No influence of the impact loading on the transformation temperatures was detected for $\beta_{1} \rightarrow \gamma_{1}{ }^{\prime} \mathrm{MT}$, in contrast to elastic properties of the martensitic phase, indicating that structural changes due to the high-velocity impact do not affect appreciably the thermoelastic equilibrium and hysteretic motion of parent - martensite boundaries during the MT.
\end{abstract}

\section{INTRODUCTION}

Performance and properties of materials exhibiting a thermoelastic martensitic transformation (TMT) are closely related to the stability of their defect structure $[1,2]$. From the engineering standpoint, the properties of TMT materials under the ultimate conditions of high-energy impact loading are of a great importance. Recently, detailed investigations have been performed of the influence of the high-velocity impact loading on the performance and properties of $\mathrm{Cu}-\mathrm{Al}-\mathrm{Ni}$ single crystals of different compositions, both in the $\beta_{1}{ }^{\prime}$ martensite [3-5] and in the $\beta_{1}$ phase $[4,6]$. An acoustic technique has been used extensively to detect the structural changes induced by the shock-wave loading of $\mathrm{Cu}-\mathrm{Al}-\mathrm{Ni}$ crystals. The present paper reports the influence of high-velocity impact on the elastic and anelastic properties of the $\beta_{1}$ and $\gamma_{1}{ }^{\prime}$ phases and on the $\beta_{1} \rightarrow \gamma_{1}{ }^{\prime}$ transformation temperatures. An estimate of the upper time limit to induce the TMT and plastic deformation of the martensitic phase is also obtained.

\section{EXPERIMENTAL}

Samples for the investigations were prepared from Cu-14.4wt\%Al-5.5wt\% Ni single crystalline rods with [100] orientation in the $\beta_{1}$ phase. Crystals were quenched into water at room temperature after homogenization at $1173 \mathrm{~K}$ for $15 \mathrm{~min}$. The $\beta_{1} \rightarrow \gamma_{1}{ }^{\prime}$ transformation was temperature-induced on cooling of crystals and stress-induced during deformation at room temperature. For impact loading, rod-shaped samples with dimensions of about $1 \times 3 \times 30 \mathrm{~mm}^{3}$ were spark-cut along the crystals.

Shock-wave loading was performed by means of a striker accelerated in a gas gun to velocities in the range of $35-500 \mathrm{~m} / \mathrm{s}$ [7]. Samples were embedded in a massive brass holder and a uniaxial plane compressive strain wave was induced in the holder with the sample. The uniaxial plane strain wave propagated along the shortest axis of the rod-shaped samples. The amplitude of the impact wave was 
characterized by the normal stress component in the direction of wave propagation, and was registered by means of tensometers and laser interferometer [7]. This stress component had a magnitude of 0.4-5.4 GPa with a pulse duration of about $2 \times 10^{-6} \mathrm{~s}$. The samples were impact loaded at room temperature.

Samples with dimensions of about $1 \times 1 \times 9.5 \mathrm{~mm}^{3}$ were spark cut from the central parts of the impacted samples for measurements of the internal friction (IF) and the Young's modulus (YM) by means of the resonant piezoelectric composite oscillator technique at a frequency of about $100 \mathrm{kHz}$. The experimental setup [8] enabled us to measure the strain amplitude dependence (strain amplitude ranged from $2 \times 10^{-7}$ to $2 \times 10^{-4}$ ) and temperature dependence (for temperatures of 90-300 K) of the IF and YM of the samples. More details of the technique have been published elsewhere [9].

The transformation temperatures of the crystals as determined by Differential Scanning Calorimetry are summarized in Table 1 .

Table 1. Transformation temperatures of samples

\begin{tabular}{|l|l|l|l|l|l|}
\hline Crystal & State & $\mathrm{M}_{\mathrm{s}}, \mathrm{K}$ & $\mathrm{M}_{\mathrm{f}}, \mathrm{K}$ & $\mathrm{A}_{\mathrm{s}}, \mathrm{K}$ & $\mathrm{A}_{\mathrm{f}}, \mathrm{K}$ \\
\hline \multirow{3}{*}{ Cu-14.4wt\%Al-5.5wt\%Ni } & as-quenched & 178 & 176 & $\approx 217$ & 232 \\
\cline { 2 - 6 } & impacted, 0.5 GPa & 180 & 179 & $\approx 220$ & 232 \\
\hline \multirow{2}{*}{ impacted, 3.1 GPa } & 179 & 177 & $\approx 218$ & 232 \\
\cline { 2 - 6 } & impacted, 5.3 GPa & 178 & 177 & & \\
\hline
\end{tabular}

\section{RESULTS}

The temperature dependence of the YM for the as-quenched and impacted samples is represented in fig.1. A drastic virtually step-like change of the YM occurred during the direct $\beta_{1} \rightarrow \gamma_{1}^{\prime}$ TMT. A detailed analysis [6] allowed us to attribute this effect to the variation of elastic properties of the material rather than to the change of the geometry of samples due to the two-way memory effect.

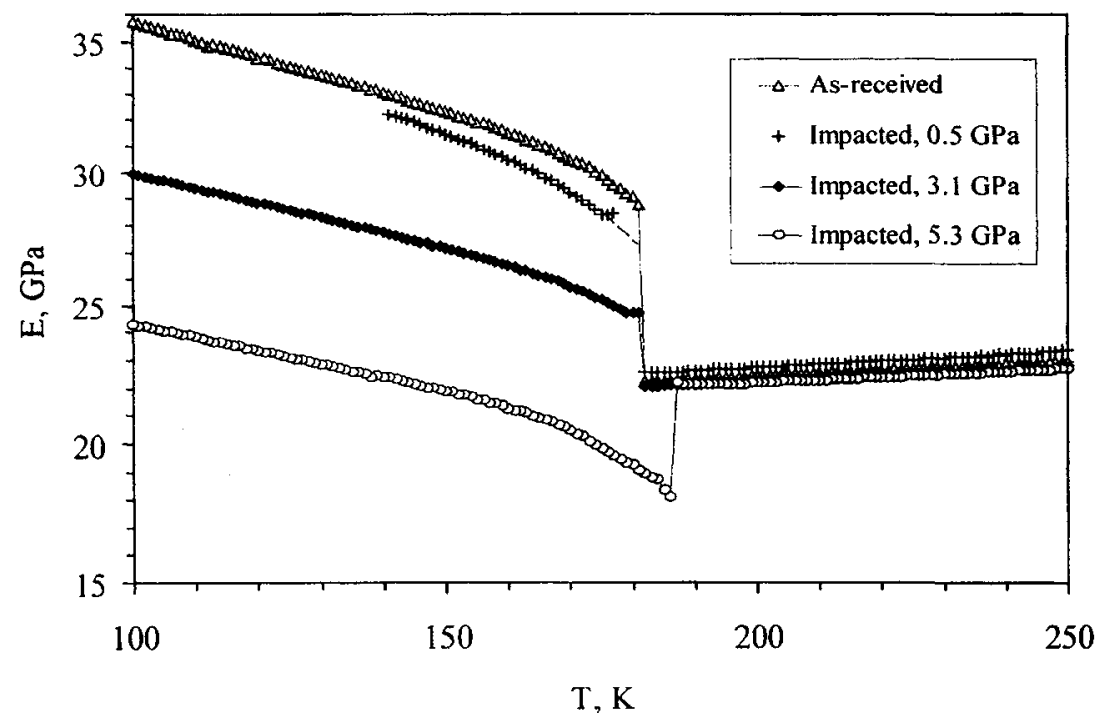

Figure I: Temperature dependence of the Young's modulus of as-quenched and impacted samples of Cu-14.4wt\%Al$5.5 \mathrm{wt} \% \mathrm{Ni}$ alloy measured during cooling at an oscillatory strain amplitude of $5 \times 10^{-7}$.

A number of other details are worth mentioning. First, impact loading of crystals in the $\beta_{1}$ phase does not lead to a detectable change of the YM in the austenite. Second, in line with the DSC 
measurements, the impact loading has only a marginal influence on the $\mathbf{M}_{\mathbf{s}}$ temperature as detected by acoustic measurements, within a few degrees. Third, despite the dramatic shift of the YM in the martensite to lower values with the increase of the impact amplitude, the temperature trend is preserved. In contrast to the $\beta_{{ }^{\prime}}$ martensite [3-5,8], no anomaly is detected in the YM temperature dependence.

Figure 2 shows the IF stress amplitude dependence measured for as-quenched and impacted samples in the $\beta_{1}$ phase at room temperature and in the $\gamma_{1}{ }^{\prime}$ martensite at $\sim 100 \mathrm{~K}$. The stress amplitude $\sigma_{\mathrm{m}}$ was recalculated from the strain amplitude $\varepsilon_{m}$ measured experimentally:

$\sigma_{\mathrm{m}}=\mathrm{E}\left(\varepsilon_{\mathrm{m}}\right) \times \varepsilon_{\mathrm{m}}$. The IF in the $\beta_{\mathrm{l}}$ phase is very low and no strain amplitude dependence can be detected both for as-quenched and for impacted samples. In the martensitic state, a pronounced stress amplitude dependence is observed for as-quenched and impacted samples. The possible effect of the impact loading on the ADIF cannot be discerned reliably, fig. 2 . The fact that the IF does not exhibit a significant change after the impact loading suggests that the anelastic contribution to the change of the YM after impact loading is not the controlling factor for the $\gamma_{1}{ }^{\prime}$ martensite.

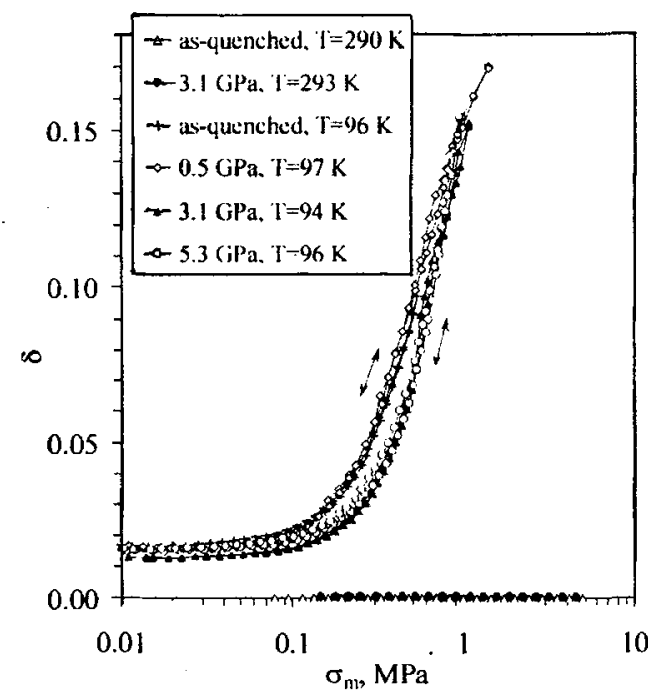

Figure 2: Stress amplitude dependence of the decrement at temperatures of about $95 \mathrm{~K}$ for asquenched and impacted samples in the $\gamma_{i}{ }^{i}$ phase.

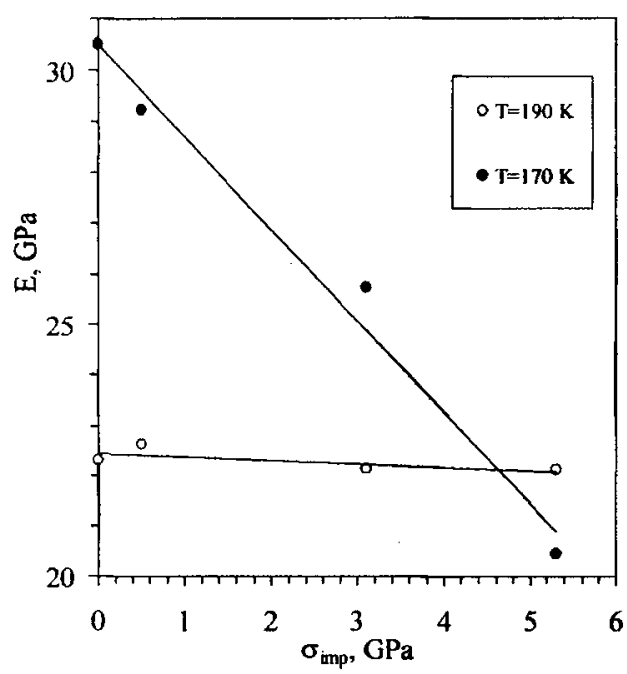

Figure 3: Influence of the impact stress on the Young's modulus of samples in the austenite $(T=190 \mathrm{~K})$ and in the martensite $(T=170 \mathrm{~K})$.

Another conspicuous detail is the absence of the amplitude hysteresis in the stress anmplitude dependence of the IF. This is contrary to what has been observed for the $\beta_{1}{ }^{\prime}$ martensite in Cu-Al-Ni $[9,10]$ and $\mathrm{Cu}-\mathrm{Al}-\mathrm{Be}$ systems [11], and points to the absence of mobile pinning points in the $\mathrm{Cu}-\mathrm{Al}-\mathrm{Ni} \gamma_{1}{ }^{\prime}$ martensite at a temperature of about $100 \mathrm{~K}$.

The dependence of the $Y M$ in the austenite and in the martensite close to $M_{s}$ and $M_{r}$, respectively, on the stress pulse magnitude is depicted in fig. 3. The YM in the martensite exhibits a nearly linear dependence on the impact stress amplitude, whereas, as mentioned before, the YM in the austenite is practically insensitive to the impact loading. It is worthwhile to note that the effect of the impact on the YM emerges at impact amplitudes with the stress components comparable with the static stress required to initiate the reorientation of martensitic variants $(0.25 \mathrm{GPa})$. 


\section{DISCUSSION}

For all impact amplitudes, the effect of the shock-wave loading on the transformation temperatures cannot be detected reliably, being comparable with the precision of the DSC measurements. The transformation temperatures determined by DSC correlate well with the results of the acoustic measurements. A discrepancy of about a few degrees between the data of calorimetry tests and acoustic measurements can be discerned in the $M_{s}$ and $M_{f}$ temperatures. This difference can be attributed to the difference in the geometry and size of the samples for calorimetry and acoustic measurements. We should also mention that the interval of the direct transformation is very narrow, typically about 2 degrees, see Table 1 . This is an indication that only a few martensitic variants are formed during the direct TMT. The stability of the temperatures, interval and hysteresis of the transformation indicates that the impact does not alter significantly the thermoelastic equilibrium during the TMT and hysteretic effects impeding motion of parent-martensite boundaries. Thus, we conclude that the structure of the temperature-induced martensitic variants (number of variants and their accomodation) as well as basic defects, impeding the motion of parent-martensite boundaries, are not influenced significantly by the impact loading.

The present results show that, as usually $[12,13]$, the anelasticity of the austenite is very low compared to that of the martensite. Since neither elastic nor anelastic properties of the $\beta_{1}$ phase are influenced notably by the impact loading, the behaviour of the martensite is of a prime interest. Figure 2 shows that the impact loading does not have a pronounced effect on the anelastic properties of the $\gamma_{1}{ }^{\prime}$ martensite, in contrast to the YM. A high contribution of anelastic strain to the measured YM of the $\beta_{1}{ }^{\prime}$ martensitic phase was detected as an anomaly of its temperature dependence [3-5,9]. Figure 1 demonstrates that, in line with the relative stability of the strain amplitude dependence of the IF, the anomaly of the YM temperature dependence is not observed in the as-quenched and impacted $\gamma_{1}{ }^{\prime}$ martensite. Moreover, the trend of the YM temperature dependence is not influenced by the impact. Therefore, it is reasonable to suggest that a possible contribution of anelastic effects to the YM does not change appreciably with impact amplitude. This conclusion is contrary to what has been reported for the $\beta_{1}{ }^{\prime}$ martensite $[4,5]$. This difference can be associated with the presence and the absence of mobile quenched-in pinning points in the $\beta_{1}{ }^{\prime}$ and $\gamma_{1}{ }^{\prime}$ martensites, respectively. The absence of mobile pinning points in the $\gamma_{1}^{\prime}$ martensite (at least at temperatures of about $100 \mathrm{~K}$ ) is evidenced by the absence of the amplitude hysteresis, see fig. 2. As has been shown, interaction of mobile partial dislocations with atmospheres of quenched-in defects controls to a great extent the anelasticity of the $\beta_{1}{ }^{\prime}$ martensite for the $\mathrm{Cu}-\mathrm{Al}-\mathrm{Ni}[3-5,9,10,14]$ and $\mathrm{Cu}-\mathrm{Al}-\mathrm{Be}[11]$ systems. Most likely, quenched-in defects for the present alloy composition are annealed out in the $\beta_{1}$ phase at room temperature. The stability of the anelastic properties of the $\gamma_{i}^{\prime}$ martensite after the impact enables us to ascribe the influence of the impact on the YM mostly to elastic effects.

The effect of the impact on clastic properties of martensite can be interpreted based on the concept of internal stresses, which are known to influence strongly the $\mathrm{YM}$ and anelastic properties of martensitic phases [3-5]. We propose the following sequence of events, leading to the sensitivity of the YM in the martensite to the impact loading of the $\beta_{1}$ phase.

- The stress-induced TMT occurs under the action of the impact with a duration of about $2 \times 10^{-6} \mathrm{~s}$.

- In addition to the TMT, the impact induces plastic deformation of the $\gamma_{1}$ martensitic phase through the motion of intervariant boundaries. Just as in the case of impact loading of the $\beta_{1}{ }^{\prime}$ martensite [3-5], high internal stresses are generated by the plastic deformation of the polyvariant martensitic structure due to high elastic anisotropy of the martensitic phase.

- The reverse TMT occurs with the completion of the action of the stress pulse. However, a change of the pattern of structural defects and internal stresses, induced in the martensite, is inherited by the single crystal of austenite, most likely through its dislocation structure.

- During subsequent direct TMT under thermal cycling, the internal stress pattern created by the impact loading governs the structure of martenitic variants nucleated during the TMT. The formation of variants with a preferred orientation leads to a significant change of the effective YM of samples, due to the high elastic anisotropy of the $\gamma_{1}{ }^{\prime}$ phase.

Thus, fundamentally this effect is similar to the two-way memory effect. However, the registered effect in the elastic properties is at least an order of magnitude higher than that expected from the change 
of the geometry of the samples. The suggested concept implies that strong anisotropy of the YM in the $\gamma_{1}{ }^{\prime}$ martensitic phase exists. To determine the orientation dependence of the YM we used the values of the 9 independent elastic constants $c_{y}$ reported in ref. [15]. First, the reciprocal matrix of the elastic compliances $s_{i j}$ was calculated from the data of ref. [15]. Then, the inverse YM in the direction of the unit vector $l_{i}$ can be calculated from the elastic compliances [16]:

$$
\frac{l}{E}=l_{1}^{4} s_{11}+2 l_{1}^{2} l_{2}^{2} s_{12}+2 l_{1}^{2} l_{2}^{2} s_{13}+l_{2}^{4} s_{22}+2 l_{2}^{2} l_{3}^{2} s_{23}+l_{3}^{4} s_{33}+l_{2}^{2} l_{3}^{2} s_{44}+l_{1}^{2} l_{3}^{2} s_{55}+l_{1}^{2} l_{2}^{2} s_{44} \text {. }
$$

Figure 4 shows the calculated orientation dependence of the $Y M$ of the $\gamma_{1}{ }^{\prime}$ martensitic single crystal. The YM attains the lowest value of about $20 \mathrm{GPa}$ in the [010] direction. A maximum of the YM of about 160 $\mathrm{GPa}$ is expected in the direction [430]. The exact comparison of measured (fig. 1) and calculated values of the YM (fig. 4) is difficult since measurements in the present work and in ref. [15] were performed at different temperatures and the softening due to the TMT should be also accounted for. Nevertheless, quite

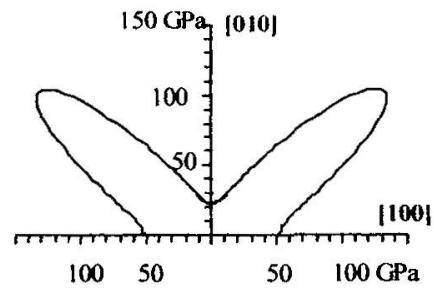

(a)

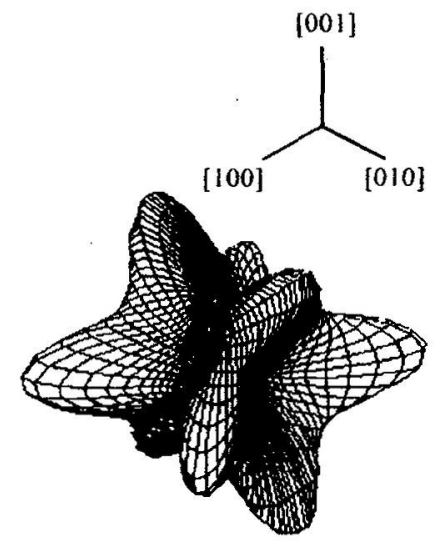

(d)

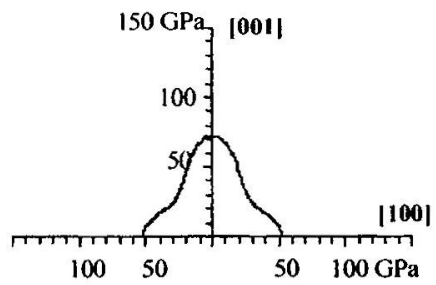

(b)

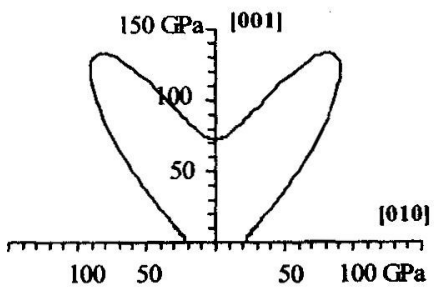

(c)

Figure 4: Orientation dependence of the Young's modulus of the $\gamma_{1}^{\prime}$ martensite: (a), (b), (c) - three cross-sections of the Young's modulus field (d).

low experimentally measured values of the YM indicate that even in the as-quenched state a preferential orientation of martensitic variants exists close to the [010] direction along the samples. It appears that after the impact loading the martensitic variants with the lowest YM are even better aligned along the impacted crystal. The high anisotropy of the the YM of the $\gamma_{1}^{\prime}$ martensite accounts thus for the virtual discrepancy in the behaviour of the YM and the transformation temperatures: minor changes in the variant structure are sufficient to change dramatically the YM, while the translormation temperatures are practically not influenced by the impact.

\section{SUMMARY}

1. The present results indicate that the stress-induced $\beta_{1} \rightarrow \gamma_{1}{ }^{\prime}$ martensitic transformation occurs for a stress pulse duration of $2 \times 10^{-6} \mathrm{~s}$. This time interval appears to be sufficient also for the subsequent deformation of the $\gamma_{1}{ }^{\prime}$ martensitic phase to occur. 
2. A structure memory effect has been found: $\mathrm{Cu}-\mathrm{Al}-\mathrm{Ni}$ austenitic crystals, shock-loaded at room temperature to induce $\gamma_{1}{ }^{\prime}$-martensite, recall during consequent temperature-induced martensitic transformation the martensitic variant structure (elastic properties) formed under the shock loading.

3. Elastic properties of $\gamma_{1}{ }^{\prime}$ crystals of the $\mathrm{Cu}-\mathrm{Al}-\mathrm{Ni}$ system are extremely sensitive to the shockwave loading. Mechanisms of this effect includes the generation of internal stresses due to the high elastic anisotropy of the martensitic phase. These internal stresses govern the formation of the martensitic variant structure during the temperature-induced martensitic transformation.

4. In contrast to elastic properties, the transformation temperatures, the interval and hysteresis of the thermoelastic martensitic transformation are insensitive to the impact loading, indicating that the impact does not alter significantly the thermoelastic equilibrium during the TMT and hysteretic effects impeding the motion of parent-martensite boundaries.

\section{Acknowledgements}

The work was supported through INTAS project No. 96-2142.

\section{References}

1. J. Van Humbeeck and R. Stalmans, in: Shape memory materials, K. Otsuka and C.M. Wayman, Eds., Cambridge University Press, UK, 1998, p. 149.

2. J. Van Humbeeck, M. Chandrasekaran, and L. Delaey, ISIJ International, 29, 388(1989).

3. Yu.Emel'yanov, S.Golyandin, N.Kobelev, S.Kustov, S.Nikanorov, G.Pugachev, K.Sapozhnikov, A.Sinani, Ya.Soifer, J.Van Humbeeck, and R.De Batist, Proc. Intern. Conf. on Internal Friction and Ultrasonic Attenuation in Solids (ICIFUAS-12, Buenos Aires, 1999), to be published in J. Alloys Comp.

4. Yu. Emel'yanov, S. Golyandin, S.Kustov, S. Nikanorov, G. Pugachev, K. Sapozhnikov, A. Sinani, J. Van Humbeeck, R. Schaller and R. De Batist, Scripta Materialia, in print.

5. S. Golyandin, S. Kustov, S. Nikanorov, K. Sapozhnikov, A. Sinani, J. Van Humbeeck, R. Schaller and R. De Batist, Acta Materialia, submitted.

6. S. Golyandin, S. Kustov, S. Nikanorov, K. Sapozhnikov, A. Sinani, J. Van Humbeeck, R. Schaller and R. De Batist, Acta Materialia, submitted.

7. N.A. Zlatin, S.M. Mochalov, G.S. Pugachev, and A.M. Bragov, Sov. Phys. Tech. Phys. 20, 428(1975).

8. S.B. Kustov, B.K. Kardashev, V.I. Ivanov, S.N. Golyandin, Yu.A. Burenkov, S.P. Nikanorov, V.M. Chernov, H. Lüft, G. Mattausch, E. Hegenbart, and Y. Schreiber, Preprint FEI-2141, (FEI, Obninsk, 1990). (In Russian).

9. S. Kustov, S. Golyandin. K. Sapozhnikov, J. Van Humbeeck, and R. De Batist, Acta mater., 46, 5117 (1998).

10. K. Sapozhnikov, S. Golyandin, S. Kustov, J. Van Humbeeck, and R. De Batist, Acta mater., 48, 1141 $(2000)$.

11. S. Kustov, S. Golyandin, K. Sapozhnikov, and M. Morin, Scripta Mater., in print.

12. R. De Batist, in V.K.Kinra and A.Wolfenden (eds.), M3D: Mechanics and Mechanisms of Material Damping. ASTM STP No.1169, ASTM, Philadelphia, 1992, p.45.

13. J. Van Humbeeck, J. Stoiber, L. Delaey and R. Gotthardt, Zs. Metallkunde, 86, 176 (1995).

14. S.B. Kustov, S.N. Golyandin. I. Hurtado, J.Van Humbeeck, and R. De Batist, M3D III: Mechanics and Mechanisms of Material Damping, ASTM STP 1304 (ed. by A. Wolfenden and V.K. Kinra), p. 94, ASTM, Philadelphia, 1997.

15. M. Yasunaga, Y. Funatsu, S. Kojima, K. Otsuka, and T. Suzuki, Scripta metall., 17, 1091(1983).

16. M.A. Nye, Physical properties of crystals. Oxford University Press, London, 1957, p. 145. 